\title{
ANÁLISIS PRELIMINAR DE ÁCIDOS GRASOS RECUPERADOS DE CERÁMICAS ARQUEOLÓGICAS DEL CURSO INFERIOR DEL RÍO COLORADO (NORPATAGONIA ORIENTAL): APORTES PARA LA SUBSISTENCIA DE GRUPOS CAZADORES-RECOLECTORES
}

\author{
LUCIANA STOESSEL", GUSTAVO MARTÍNEZ"* \& DIANA CONSTENLA
}

\begin{abstract}
RESUMEN
En este trabajo se presentan los resultados preliminares de los análisis de ácidos grasos realizados en tiestos cerámicos recuperados de tres sitios arqueológicos del curso inferior del río Colorado (Norpatagonia oriental, Argentina). Los contextos arqueológicos corresponden al Holoceno tardío, tanto inicial (ca. 3.000-1.000 años AP) como final (ca. 1.000-250 años AP). La información obtenida sugiere la cocción de grandes herbívoros y posiblemente de plantas y/o médula ósea, así como la presencia de recursos de origen marino, muy probablemente peces. En términos generales, estos resultados son consistentes con las principales tendencias descriptas en el modelo de subsistencia propuesto para el área, generado desde los estudios zooarqueológicos y de los análisis de isótopos estables en restos óseos humanos. Además, los resultados obtenidos de los análisis de ácidos grasos permitieron inferir aspectos relacionados con los lugares específicos de explotación y consumo de ciertos recursos (i.e., peces) y estrategias de movilidad y cobertura del paisaje por parte de los grupos cazadores-recolectores del Holoceno tardío.
\end{abstract}

PALABRAS CLAVE: ácidos grasos, Norpatagonia, subsistencia, Holoceno tardío

PRELIMINARY ANALYSIS OF FATTY ACIDS RECOVERED FROM ARCHAEOLOGICAL POTTERY OF THE LOWER COURSE OF THE COLORADO RIVER (NORTH-EASTERN PATAGONIA): CONTRIBUTIONS TO THE HUNTER-GATHERERS SUBSISTENCE PATTERNS

\section{ABSTRACT}

This paper presents the preliminary results obtained of the analysis of fatty acids recovered from pottery sherds from three archaeological sites of the lower course of the Colorado River (North-eastern Patagonia, Argentina). These sites correspond chronologically to the late Holocene, both initial (ca.

INCUAPA-CONICET. FACSO-UNCPBA. Avda. del Valle 5737, Olavarría (B7400JWI), Argentina.

lstoesse@soc.unicen.edu.ar

* INCUAPA-CONICET. FACSO-UNCPBA. Avda. del Valle 5737, Olavarría (B7400JWI), Argentina.

gmartine@soc.unicen.edu.ar

PLAPIQUI. UNS-CONICET. Camino La Carrindanga km 7, Bahía Blanca (8000), Argentina. dconstenla@plapiqui.edu.ar 
3.000-1.000 years BP) as final (ca. 1.000-250 years BP) periods. The information obtained suggests cooking of large herbivores and possibly plants and/or bone marrow, as well as the presence of marine resources, most likely fish. In general terms, these results are consistent with the main trends described in the subsistence model proposed for the area, from zooarchaeological evidence and information provided by stable isotopes from bone human remains. In addition, results of fatty acids allowed inferring aspects related to the exploitation and consumption of certain resources (i.e., fish) at specific places of landscape (Atlantic coast) as well as mobility strategies by late Holocene hunter-gatherer groups.

KEY WORDS: fatty acids, Northern Patagonia, subsistence, late Holocene.

\section{INTRODUCCION}

El estudio de los ácidos grasos preservados en las paredes de las vasijas se ha convertido en una importante vía de análisis para evaluar aspectos vinculados con el uso de las mismas, en particular aquellos relacionados a las prácticas asociadas con las actividades culinarias (i.e., almacenamiento, cocción, hervido). Asimismo, las proporciones en las que se encuentran los distintos ácidos brindan información con respecto a las clases de alimentos que han sido almacenados o cocinados en los recipientes cerámicos (Buonasera, 2005; Charters et al. 1995; Eerkens, 2005, 2007; Evershed et al. 1997, 1999; Malainey, 2007; Malainey et al. 1999a, 1999b, 1999c; Spangenberg et al. 2006). Si bien este enfoque no permite realizar determinaciones de taxones específicos, posibilita identificar categorías amplias de recursos (i.e., mamíferos terrestres, peces, plantas, etc.) y puede constituir una primera aproximación para discutir el rol de los mismos sobre todo en aquellos sitios en los cuales los restos faunísticos $y$ vegetales no se preservaron (Evershed et al. 1997; Isaksson, 2009). En este trabajo se aborda esta línea de investigación y se presentan los primeros resultados obtenidos de los análisis de ácidos grasos realizados en tiestos cerámicos recuperados en distintos sitios del curso inferior del río Colorado (pcia. Buenos Aires; Fig. 1). Con esta información se busca complementar y poner a prueba los resultados obtenidos a partir de los análisis faunísticos e isotópicos generados para el área de estudio (Alcaraz, 2012; Martínez et al. 2009a; Stoessel, 2012). En este sentido, para el Holoceno tardío, se propuso un modelo de subsistencia a partir de los análisis zooarqueológicos realizados en diversos sitios.
El mismo señala que la dieta estuvo basada en la explotación del guanaco, complementado con el consumo del venado y ñandú y el aporte de recursos vegetales. Este patrón es característico del Holoceno tardío inicial (ca. 3.000-1.000 años AP). Hacia el Holoceno tardío final (ca. 1.000250 años AP) se produjo una intensificación en las actividades de subsistencia asociada con una diversificación de la dieta, con la incorporación de especies de menor tamaño procedentes de diferentes ambientes (terrestres, marinos y fluviales) y un aprovechamiento más intensivo del guanaco, peces (fluviales y marinos) y vegetales. Con respecto al guanaco, se maximizó el aporte nutricional de las carcasas a través del consumo de médula y grasa ósea. En el caso de los peces, se propuso una explotación intensiva debido a la alta frecuencia de sus restos en los sitios (Alcaráz, 2012; Stoessel, 2012; Stoessel \& Martínez, 2014).

Por otro lado, la información obtenida a partir de los análisis isotópicos de restos óseos humanos provenientes de sitios del área de estudio, así como del curso medio del río Negro, indica que la subsistencia estuvo basada en la explotación de herbívoros consumidores de plantas $\mathrm{C}^{3} \mathrm{y} / \mathrm{o}$ el consumo de este tipo de vegetales durante el Holoceno tardío. Asimismo, los valores de $\delta \mathrm{N}^{15}$ muestran un enriquecimiento que se encuentra dentro del rango de los individuos que consumen especies acuáticas. A partir de estos resultados se propuso que los peces habrían sido recursos complementarios en la dieta (Martínez et al. 2009a). En términos generales, la información proveniente de los análisis isotópicos se condice con los resultados obtenidos de los análisis faunísticos. No obstante, hay hasta el momento una disparidad entre el registro zooarqueológico e 


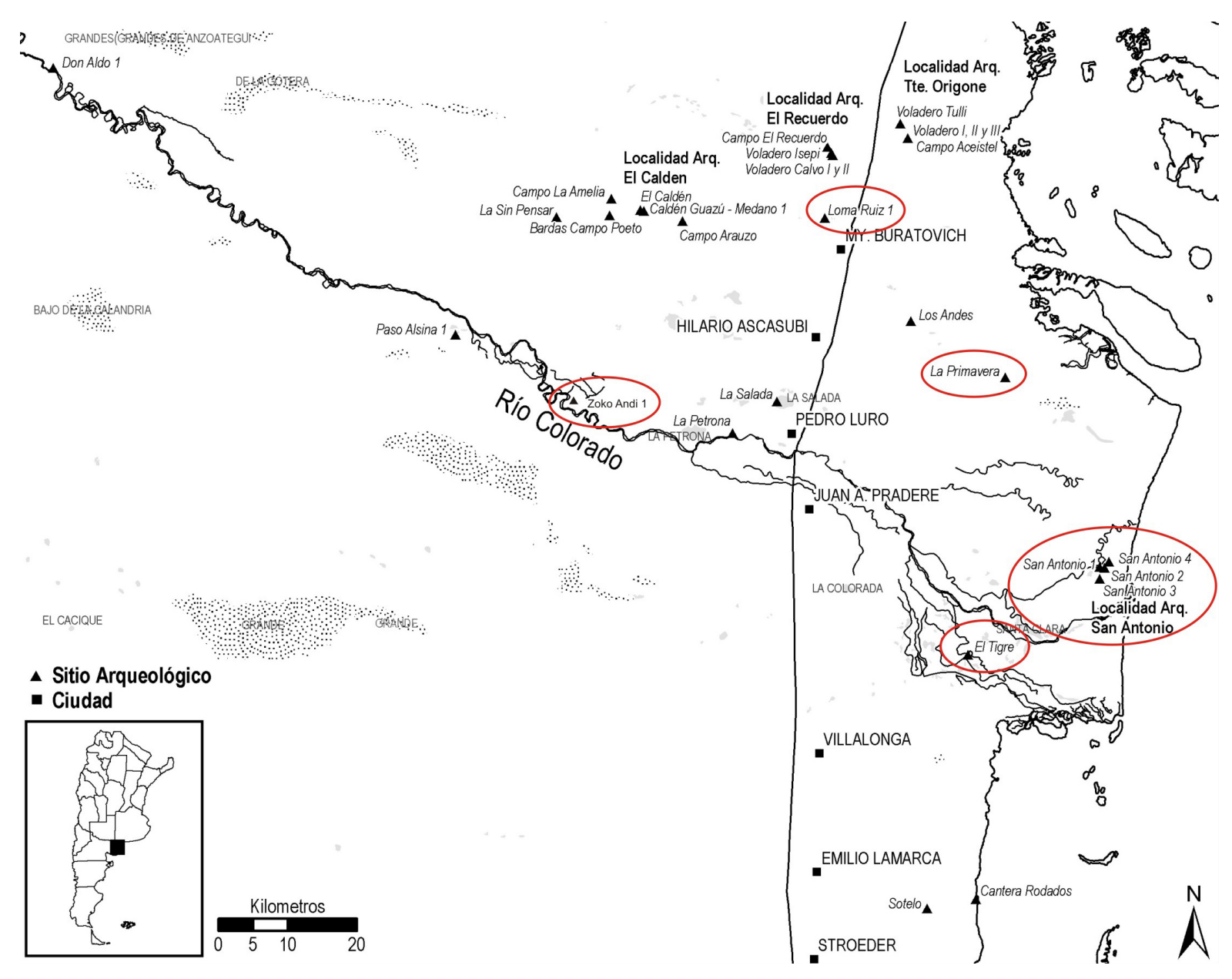

Fig. 1. Curso inferior del río Colorado. Los círculos marcan los sitios arqueológicos de los cuales se recuperaron tiestos cerámicos utilizados para el análisis de ácidos grasos y otros sitios citados en el texto.

isotópico del Holoceno tardío inicial y final ya que mientras la señal isotópica para todo el Holoceno tardío muestra a los peces como un complemento de la dieta sin cambios diacrónicos evidentes, el registro arqueológico indica un mayor consumo durante el Holoceno tardío final (ver discusión en Stoessel, 2012). En consecuencia, el objetivo de este trabajo es realizar una primera aproximación al estudio de los ácidos grasos provenientes de cerámicas arqueológicas recuperadas en el área de estudio con el fin de evaluar si la información obtenida de dichos ácidos es concordante con los cambios propuestos durante el Holoceno tardío en las dietas y la subsistencia a partir del registro zooarqueológico e isotópico. En otras palabras, se utilizará a los ácidos grasos como una línea independiente de análisis que permita discutir algunos aspectos del modelo de subsistencia propuesto.

\section{APLICACIÓN DEL ESTUDIO DE ÁCIDOS GRASOS EN EL ANÁLISIS DE MUESTRAS ARQUEOLÓGICAS}

Durante su uso la vasija absorbe distintos componentes orgánicos de los recursos que están siendo almacenados o procesados en ella. Los análisis arqueológicos se han centrado en general en el estudio de los ácidos grasos debido a que son moléculas relativamente estables y por lo tanto menos afectadas por la degradación post-depositacional. Sin embargo, la exposición 
a altas temperaturas como consecuencia de la cocción puede generar la degradación de estos elementos. Si bien los lípidos son más resistentes a la descomposición que otros componentes (i.e., carbohidratos), procesos como la oxidación y la hidrólisis pueden acelerar su degradación. En este caso, la descomposición depende de diferentes factores como la temperatura, la disponibilidad de oxígeno y agua, así como también de aspectos relacionados con el contexto de depositación (Eerkens, 2005, 2007; Evershed et al. 2002; Koirala \& Rosentreter, 2009). Asimismo, se debe considerar que la degradación puede modificar el porcentaje relativo en el que se encuentran representados los distintos ácidos grasos en una muestra debido a que la descomposición no es uniforme. En este sentido, los ácidos insaturados (aquellos que poseen enlaces dobles entre átomos de carbón) se degradan más rápidamente que los saturados (aquellos que no poseen enlaces dobles entre átomos de carbón), al igual que los componentes de cadenas largas en comparación a los de cadenas cortas. Esta situación complejiza las comparaciones directas entre los perfiles de ácidos grasos obtenidos de los materiales arqueológicos $y$ aquellos procedentes de recursos actuales no sometidos a cocción (Buonasera, 2005; Eerkens, 2005, 2007; Malainey et al. 1999a).

La problemática descripta anteriormente generó el desarrollo de trabajos experimentales con el fin de obtener patrones en la descomposición de ácidos grasos que permitan compararlos con los provenientes de muestras arqueológicas y, de esta manera identificar con un menor grado de ambigüedad los recursos que han sido cocinados en las vasijas (Eerkens, 2005; Evershed et al. 2008; Frère et al. 2010; Malainey et al. 1999a). Malainey et al. (1999a) analizaron los cambios en la composición de los ácidos grasos conservados en las paredes de vasijas experimentales en las que se cocinaron distintos recursos (herbívoros, peces y plantas). Estos autores observaron que si bien la degradación tuvo efectos en la composición de los ácidos grasos, las proporciones en las que se encontraron permiten diferenciar categorias amplias de recursos. A partir de las tendencias obtenidas, establecieron una serie de criterios para identificar las distintas clases de recursos en vasijas arqueológicas (ver Tabla 4 en Malainey et al. 1999a). Eerkens (2005), por su parte, teniendo en cuenta los resultados obtenidos por Malainey et al. (1999a) estableció 4 índices (C12:0/C14:0; C16:0/C18:0; C16:1/C18:1; C15:0+C17:0/ C18:0) que comprenden 8 ácidos grasos comúnmente recuperados en tiestos arqueológicos. Estos índices, que expresan relaciones entre éstos ácidos permiten diferenciar 5 clases de recursos (mamíferos terrestres, peces, semillas, bayas, raíces y "greens"; Eerkens, 2005, 2007). Asimismo, se han generado índices para distinguir aquellos recursos que no fueron sometidos a cocción. Entre ellos, Marchbanks (1989) propone un método que se basa en el cálculo de un coeficiente (C12:0+C14:0/C12:0+C14:0+C18:2+C18:3) que permitiría discriminar entre plantas, mamíferos terrestres y peces (ver Buonasera, 2005; Malainey et al. 1999a).

En Argentina, Frère et al. (2010) llevaron a cabo un trabajo experimental con el objetivo de caracterizar los ácidos grasos de cuatro especies (guanaco, coipo, vizcacha y bagre) y evaluar las transformaciones que sufren los mismos al ser sometidos a cocción. En esta experimentación, hirvieron en eventos distintos carne de vizcacha y carne de coipo, y en un mismo evento carne de bagre y de coipo. Posteriormente, se compararon los ácidos grasos presentes en la carne cruda, cocida, en el caldo de cocción y en los conservados en las paredes de las vasijas experimentales (ver Tablas 2, 3, 4 y 5 en Frère et al. 2010). Tanto en estas últimas, así como en la carne cocida y en el caldo resultante de la cocción se registraron los ácidos grasos más representativos de los recursos hervidos (i.e., C18:0, C16:0; C18:1; C18:2n6, etc.). En este caso, si bien hay una mayor proporción de ácidos saturados en comparación a los poliinsaturados, como consecuencia de la degradación de estos últimos, la presencia de estos ácidos permitió identificar categorías de alimentos. Estos resultados son importantes ya que pueden ser utilizados como un marco de referencia para el análisis de ácidos grasos en materiales arqueológicos, teniendo en cuenta que las especies utilizadas son algunas de las más frecuentes en los conjuntos arqueológicos de la región pampeana durante el Holoceno tardío.

Lo desarrollado en este apartado muestra la complejidad en la utilización de los ácidos grasos 
como una vía de análisis para discutir aspectos relacionados con la dieta. En este sentido, se deben considerar los alcances y limitaciones de este tipo de evidencia y la escala en la que se realizan las inferencias. No obstante, si bien a partir de estos estudios no se pueden efectuar determinaciones específicas sobre los taxones preparados en las vasijas, permiten discriminar categorías generales de recursos que fueron sometidos a cocción o almacenamiento. Desde el punto de vista arqueológico, esta información constituye un importante punto de partida para abordar el estudio de prácticas culinarias y cuestiones relacionadas con la funcionalidad de las vasijas, en especial aquellas vinculadas con la cocción y/o almacenamiento de recursos. En este contexto, los índices descriptos anteriormente son un marco de referencia útil con el cual comparar los perfiles de ácidos grasos obtenidos de los tiestos arqueológicos y serán considerados para interpretar los resultados de los análisis llevados a cabo como parte de este trabajo.

\section{ANTECEDENTES SOBRE LOS ESTUDIOS DE ÁCIDOS GRASOS EN LAS REGIONES PAMPEANA Y PATAGÓNICA}

En las regiones pampeana y patagónica los análisis de ácidos grasos vinculados al abordaje de problemáticas arqueológicas comenzaron a desarrollarse recién en la última década. Estos trabajos fueron realizados sobre distintos materiales y para evaluar diversas temáticas. En la primera de las regiones mencionadas, se están llevando a cabo estudios en esta línea de evidencia en varias áreas. En el sector del Pre-Delta del río Paraná, Bonomo et al. (2012) realizaron análisis de ácidos grasos y de restos botánicos en una pieza cerámica (cuchara) procedente del sitio Cerro Tapera Vázquez con el fin de determinar la función para la que fue utilizada. Los resultados obtenidos indican que la cuchara habría contenido recursos vegetales, posiblemente aceites de semillas o ceras (Bonomo et al. 2012). En el área del humedal del río Paraná inferior se llevaron a cabo análisis de ácidos grasos de tiestos cerámicos procedentes de diversos sitios con el objetivo de analizar el rol de la cerámica en el procesamiento de recursos y las conductas alimentarias de los grupos que habitaron esta área. Las tendencias obtenidas de los ácidos grasos son consistentes con los resultados zooarqueológicos y sugieren un énfasis en el procesamiento y consumo de peces y vegetales (Pérez et al. 2013). Para el área del litoral fluvial bonaerense (estuario del Río de la Plata), Pérez Meroni et al. (2010) utilizaron el análisis de ácidos grasos como parte de los estudios destinados a evaluar el procesamiento y/o aprovechamiento de vegetales. Los análisis realizados en fragmentos cerámicos de un sitio (sitio Las Marías) mostraron la presencia de ácidos grasos cuya composición sugiere el posible procesamiento de pescado y de maíz (Pérez Meroni et al. 2010).

En la Depresión del Río Salado, se incorporó el análisis de ácidos grasos como línea de evidencia. En este sentido, González de Bonaveri y Frère (2002) y González (2005) realizaron análisis de ácidos grasos en fragmentos cerámicos arqueológicos, pigmentos y tiestos provenientes de recipientes experimentales para evaluar el uso de las vasijas. Los resultados de estos trabajos les permitieron determinar que los fragmentos de alfarería fueron parte de vasijas utilizadas para la cocción o almacenamiento de productos, así como inferir los posibles recursos preparados en ella. En este sentido, la composición de los ácidos sugiere la cocción de herbívoros terrestres de tamaño mediano (coipo), posiblemente peces y vegetales (González de Bonaveri \& Frère, 2002).

En otras áreas de la región, los análisis de ácidos grasos fueron realizados también en otro tipo de materiales. En este sentido, Babot et al. (2007) llevaron a cabo estudios de los restos microfósiles y de residuos orgánicos recuperados en los materiales de molienda provenientes de sitios cercanos a la costa (El Guanaco 1 y 2) y del área de Tandilia (La China 1) con el fin de evaluar el uso de los mismos. En todas las muestras se recuperaron ácidos grasos, cuya composición sugiere la presencia de residuos vinculados al procesamiento de plantas $\mathrm{y}$, posiblemente, recursos cárneos (Babot et al. 2007). En esta misma área, Mazzia (2010) incorporó el análisis de ácidos grasos al estudio del material lítico proveniente de varios sitios (Cueva Zoro, El Ajarafe, Cerro la China sitios 1, 2 y 3 y Cerro 
El Sombrero Cima) como una línea de evidencia para evaluar el uso de recursos que no se preservaron en esa área (Mazzia, 2010; Mazzia \& Weitzel, 2008). Como parte de este trabajo, se realizaron análisis de ácidos grasos en piezas líticas experimentales y en muestras de sedimento para determinar posibles contaminaciones. Los resultados mostraron que los artefactos fueron utilizados sobre recursos diversos. En este sentido, se detectaron ácidos grasos cuya composición es compatible con aceite de semillas y con grasas animales acuáticas (Mazzia, 2010).

En el sector oriental de las sierras de Tandilia, Di Prado et al. (2007) analizaron los pigmentos minerales recuperados en el sitio arqueológico Calera. Como parte de estos trabajos, llevaron a cabo estudios de ácidos grasos de la fracción orgánica para evaluar la posibilidad del uso de aglutinantes, ya sean de origen vegetal o animal. En este caso, pudieron identificar la presencia de ácidos grasos en dos de las muestras pero los resultados aún no son concluyentes ( $\mathrm{Di}$ Prado et al. 2007).

Para el área centro-sur de la provincia de La Pampa, Illescas et al. (2012) realizaron análisis de ácidos grasos en tiestos cerámicos provenientes de la localidad Tapera Moreira. Los resultados obtenidos sugieren el uso de vasijas para la preparación de alimentos de origen vegetal $\mathrm{y}$ animal (Illescas et al. 2012).

En la región Patagónica, Schuster (2012) llevó a cabo estudios de la tecnología cerámica en diversos sitios del área costera del noreste de Chubut con el fin de evaluar el rol que habría tenido la misma en los grupos cazadores-recolectores que habitaron esta área. Como parte de este trabajo, realizó análisis de ácidos grasos en una muestra de fragmentos que presentaban diferencias en cuanto al tratamiento de superficie, decoración, diámetro de boca y morfología. Los resultados sugieren, en todos los casos un uso doméstico generalizado $\mathrm{y} / \mathrm{o}$ multifuncional de las vasijas vinculado a la cocción de vegetales $y$, en algunos casos de proteínas y grasas terrestres y marinas. Estas últimas posiblemente correspondan a peces (Schuster, 2012; Gómez Otero et al. 2014).

En otras áreas de esta región, el análisis de ácidos grasos fue incluido en algunos trabajos vinculados al estudio del arte rupestre. En este sentido, Maier et al. (2007) analizaron los compuestos orgánicos e inorgánicos de dos muestras de ocre que fueron obtenidas en un sitio arqueológico (Cueva Loncomán) en la provincia de Río Negro con el objetivo de evaluar la composición de las pastas. Para determinar los componentes orgánicos llevaron a cabo estudios de ácidos grasos, cuyos resultados fueron comparados con la composición de ácidos de la grasa y médula de Pteronemia pennata y médula de Lama guanicoe. Los análisis de ácidos grasos confirmaron que los aglutinantes utilizados eran de origen animal y que su composición es similar a la de la grasa y médula de las especies arriba mencionadas (Maier et al. 2007).

Por otro lado, para el área del Canal de Beagle, Fiore et al. (2008) analizaron los componentes orgánicos e inorgánicos de pigmentos provenientes de tres sitios arqueológicos (Túnel 1, Shamakush 1 e Imiwaia 1) para determinar si los mismos fueron usados en su estado natural o si fueron mezclados con otros aglutinantes orgánicos. Asimismo, evaluaron la posible contaminación de las muestras mediante la comparación de los resultados obtenidos con la información de los análisis realizados en muestras de sedimentos de los niveles en los que se encontraba el material arqueológico. A partir de los datos proporcionados por los estudios de ácidos grasos estos autores sugieren que el componente orgánico en las muestras de pigmentos no fue afectado por procesos tafonómicos sino que es el resultado de acciones humanas, quizás relacionadas con la incorporación de estos compuestos como aglutinantes para la preparación de pintura (Fiore et al. 2008).

\section{MATERIALES Y MÉTODOS}

\section{Contextos de procedencia de las muestras:}

Las muestras analizadas en este trabajo provienen de tres sitios, ubicados en diferentes sectores del área de estudio (Fig. 1) y correspondientes tanto al Holoceno tardío inicial como al Holoceno tardío final. Uno de ellos es Loma Ruíz 1, cuya ocupación se ubica en la parte inicial del Holoceno tardío (ca. 1.900-1.600 años AP; Fig. 1). En este sitio 


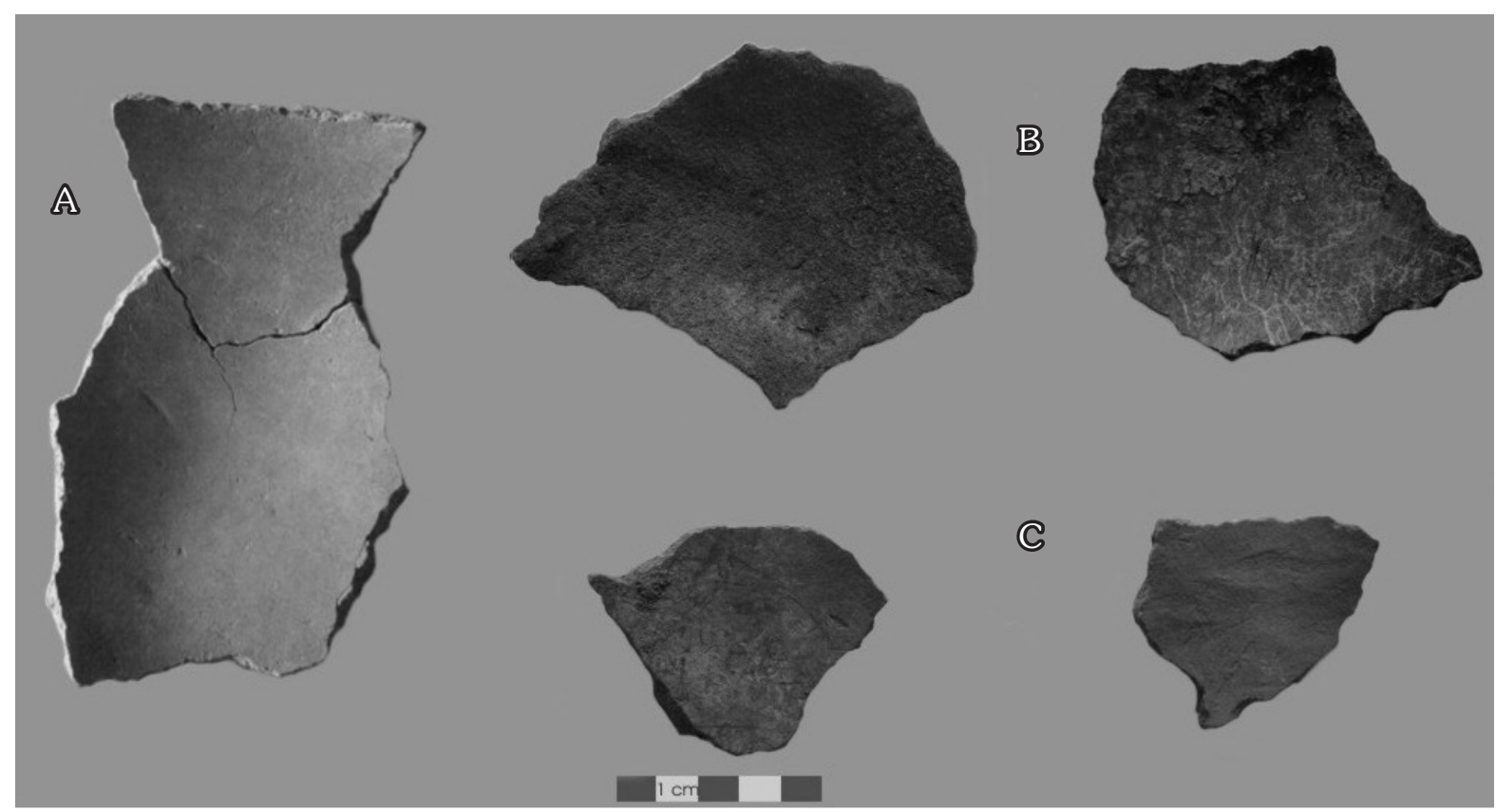

Fig. 2. Superficies internas de algunos tiestos analizados: $A=$ Loma Ruíz 1; B= El Tigre; C= Zoko Andi 1.

se recuperó una importante cantidad de restos (óseos, líticos y cerámicos) y fue caracterizado funcionalmente como una base residencial en la que se llevaron a cabo actividades domésticas a través de ocupaciones breves (Armentano, 2012; Armentano et al. 2013; Martínez et al. 2011; Stoessel, 2012). Con relación a los taxones presentes en este sitio, se identificaron restos correspondientes a especies de tamaño mayor y a fauna menor. Con respecto a las primeras, el guanaco es la que presenta la mayor frecuencia y la única con evidencias de consumo (Stoessel, 2012). En el caso de la fauna menor, el orden Rodentia es el más representado y su ingreso al registro se produjo por causas naturales. Posiblemente, los armadillos hayan sido consumidos (Alcaráz, 2012). Con respecto a la cerámica, se recuperaron fragmentos de bordes y partes de cuerpo $(\mathrm{N}=67)$ que poseen, en general alisado en la superficie interna $y$ externa. Los espesores de las paredes están comprendidos entre los 6 y $8 \mathrm{~mm}$. A partir de las relaciones de ensamblaje realizadas entre algunos de los tiestos se estableció la presencia de contenedores subglobulares o globulares con bordes directo y directo-evertido que indican formas abiertas. Los diámetros de las bocas oscilan entre los ca. 200-250 mm. La cocción de las piezas se realizó a una atmósfera no oxidante. Asimismo, un porcentaje importante de los fragmentos presenta adherencias tanto externas como internas. En el caso de las primeras, se trata de restos de hollín, mientras que las segundas fueron detectadas por la presencia de sectores ennegrecidos que corresponden a impregnaciones (adherencias) producidas por la cocción de alimentos, de modo que se propuso un uso culinario de estos recipientes (Martínez et al. 2011; Borges Vaz com pers. 2015). Las muestras analizadas en este trabajo provienen de 6 tiestos que presentan las adherencias descriptas anteriormente (Fig. 2).

Otro de los sitios analizados es El Tigre, ubicado cronológicamente en el Holoceno tardío final (ca. 900-400 años AP; Fig. 1). A partir de las distintas actividades realizadas en este sitio se recuperó una importante cantidad y diversidad de restos líticos, cerámicos y óseos. Funcionalmente, se corresponde con una base residencial de actividades múltiples que habría sido reocupada intensamente (Alcaráz, 2012; Armentano, 2012; Martínez et al. 2009b; Stoessel, 2012). El conjunto faunístico de este sitio presenta una importante diversidad de especies terrestres y fluviales. Con relación a las primeras, el guanaco 
es la especie que posee la mayor frecuencia entre los taxones de tamaño mayor y la única con claras evidencias de consumo. Asimismo, se detectaron restos correspondientes a fauna menor, entre los cuales distintas especies de mamíferos pequeños (i.e., coipo, vizcacha $y$ caviomorfos) y aves (i.e., benteveo, garcita bruja, bandurria y anatidae) presentan evidencias de explotación. Con respecto a las especies fluviales, la perca es la más representada y la única que presenta modificaciones antrópicas (Alcaráz, 2012; Stoessel, 2012). El conjunto cerámico de este sitio proviene tanto de excavación $(\mathrm{N}=379)$ como de superficie $(\mathrm{N}=765)$. Hasta el momento, se analizó el conjunto proveniente de excavación conformado por fragmentos de cuerpo $(n=361)$ y bordes $(n=18)$. Los análisis realizados indican que el alisado fue el tratamiento más aplicado tanto en las superficies externas como internas. Los espesores de las paredes oscilan entre los 4 y $6 \mathrm{~mm}$. La atmósfera de cocción más frecuente en las piezas es la no oxidante. Las relaciones de ensamblaje realizadas sugieren preferentemente perfiles simples, de formas abiertas y con bocas comprendidas entre los 100 y $120 \mathrm{~mm}$. de diámetro. Asimismo, se identificaron fragmentos de cuerpo $(n=2)$ con presencia de orificios de suspensión o reparación (Borges Vaz com pers. 2015). En este caso, también se recuperaron tiestos con rastros de hollin y adherencias internas. Para el análisis de ácidos grasos se seleccionó una muestra de 6 tiestos provenientes de excavación que presentan las características mencionadas (hollin y/o adherencias internas; Fig. 2).

Finalmente, se analizó una muestra proveniente del sitio Zoko Andi 1 (Fig. 1). En este caso, se trata del primer sitio en el área de estudio en el que se pudieron determinar dos momentos de ocupación, uno correspondiente al Holoceno tardío inicial (ca. 1.500-1.300 años AP) y otro al Holoceno tardío final (ca. 800-400 años AP). Con respecto a la funcionalidad, se corresponde con una base residencial de actividades múltiples reiteradamente ocupada, en la que se registraron además entierros humanos (Martínez et al. 2014). Los estudios parciales realizados en dos de las cuadrículas excavadas (C4 y C6) permitieron identificar diversas especies de tamaño mayor $y$ menor. El guanaco es la especie que presenta la mayor frecuencia en el conjunto de las especies de tamaño mayor, mientras que entre la fauna menor el orden Rodentia es el mejor representado. En este conjunto, se detectaron evidencias de explotación en restos correspondientes a guanaco, dasipódidos y ave indeterminada (Martínez et al. 2014). El conjunto cerámico de este sitio (ca. $\mathrm{N}=$ 270) se encuentra en proceso de análisis. Hasta el momento, se analizó una muestra $(n=171)$ compuesta por fragmentos de cuerpos y bordes provenientes de niveles que corresponden a los dos momentos de ocupación mencionados. En general, los fragmentos presentan alisado en sus dos superficies (interna y externa), con espesores de las paredes que oscilan entre los 4 y $6 \mathrm{~mm}$. La atmósfera de cocción predominante también es en este caso la no oxidante. Con respecto a la morfología de las piezas, se pudo inferir la presencia de perfiles simples asociados a bocas abiertas pero con una orientación cerrada hacia la boca, como lo sugiere el predominio de bordes invertidos. Los diámetros de las bocas están comprendidos entre los 120 y $140 \mathrm{~mm}$. Asimismo, dos fragmentos de cuerpo presentan la mitad de un orificio de suspensión o reparación (Borges Vaz com pers. 2015). Para este trabajo se tomó una muestra compuesta por cinco fragmentos que pertenecen al segundo momento de ocupación del sitio (Holoceno tardío final), uno de los cuales presenta hollín en su cara externa (Fig. 2). Los tiestos recuperados en los niveles que corresponden al Holoceno tardío inicial no pudieron ser sometidos a análisis de ácidos grasos debido al pequeño tamaño de los fragmentos.

\section{Procedimiento:}

Los análisis fueron llevados a cabo en los laboratorios de PLAPIQUI (UNS-CONICET) por uno de los autores (D.C.). En principio, se enviaron a analizar dos muestras (una de Loma Ruíz 1 y una de El Tigre) a los efectos de determinar si las mismas contenían residuos de ácidos grasos. En ambos casos se encontraron vestigios de grasas por lo que se decidió ampliar la muestra en estos sitios e incorporar aquellas provenientes del sitio Zoko Andi $1(\mathrm{~N}=5)$. La determinación del contenido de lípidos y la composición de ácidos grasos se realizó mediante los siguientes pasos: 
Extracción de los lípidos:

Las muestras para el análisis cromatográfico se extrajeron de la parte cóncava de las piezas arqueológicas, eliminando la capa superficial mediante el raspado de las mismas con un bisturí de acero inoxidable, a los efectos de prevenir potenciales contaminaciones por procesos postdepositacionales o por manipulación durante la excavación (Costa Angrisani \& Constenla, 2010; Evershed et al. 1992; Skibo, 1992). Posteriormente, con el mismo método se obtuvieron $4 \mathrm{~g}$ de muestra de toda la superficie de la pieza. El tamaño de los tiestos es variable en todos los sitios (ver Fig. 2). Para la obtención de esta cantidad se utilizó en algunos casos más de un bisturí. La extracción de los lípidos se realizó mediante la técnica de Folch (Folch et al. 1957), utilizando como solvente una mezcla cloroformo:metanol (2:1) en una proporción de 20:1 (mL:g) de solvente con respecto a la masa de muestra. La muestra y el solvente se colocaron en un erlenmeyer tapado a temperatura ambiente y se los sometió a agitación suave y continua durante 8 hs. Esta metodología es ampliamente utilizada en la extracción de grasas para el análisis de ácidos grasos poliinsaturados, ya que el método de extracción en frío y al abrigo del oxígeno, evita el deterioro de los compuestos menos estables (Costa Angrisani \& Constenla, 2010).

- Obtención de los ésteres metílicos de ácidos grasos:

Para realizar la detección cromatográfica, realizó por cromatografía gaseosa, con las

los ácidos grasos se deben esterificar. Para esto se pesaron entre 0,25 y $0,35 \mathrm{mg}$ del extracto graso obtenido de las piezas arqueológicas en un tubo de ensayo con cierre hermético. Luego, se agregaron $2 \mathrm{~mL}$ de $\mathrm{KOH}$ (hidróxido de potasio) al 10\% en metanol y se agitó en vortex vigorosamente. Se cerró el tubo bajo atmósfera de $\mathrm{N}_{2}$, se introdujo durante 45 minutos en un baño a $80{ }^{\circ} \mathrm{C} y$, por último se dejó enfriar a temperatura ambiente (AOCS, 1997; Morrison \& Smith, 1964).

Posteriormente, se extrajo el material insaponificable colocando en el tubo $2 \mathrm{~mL}$ de éter de petróleo agitando en vortex (tres veces). En este caso, la fase etérea se desechó y la fase inferior, que contiene los jabones potásicos de los ácidos grasos, se trató con $\mathrm{HCl}$ concentrado (0,5 mL agregados con el tubo sumergido en baño de hielo) para producir la liberación de los ácidos grasos esterificados. Los ésteres metílicos de los ácidos grasos se extrajeron de la mezcla por agregado de $2 \mathrm{~mL}$ de éter de petróleo (tres veces), el extracto etéreo, contenido los AG, se evaporó bajo corriente de $\mathrm{N}_{2}$ hasta sequedad. Por último, se agregaron $1,5 \mathrm{~mL}$ de solución de $\mathrm{BF}_{3}$ al $10 \%$ en metanol, calentando durante 30 minutos a 80 ${ }^{\circ} \mathrm{C} y$, finalmente se extrajeron los ésteres metílicos con éter de petróleo ( $2 \mathrm{~mL}, 3$ veces) pasándolos a un vial donde se evaporó a sequedad en atmósfera de $\mathrm{N}_{2}$, para luego retomar con $1 \mathrm{~mL}$ de hexano cromatográfico. Cada extracción se realizó por duplicado.

- Análisis cromatográfico:

La determinación de ácidos grasos se lizadas en el análisis de ácidos grasos.

Tabla 1. Condiciones cromatográficas utilizadas en el análisis de ácidos grasos.

\begin{tabular}{|c|c|}
\hline Cromatógrafo gaseoso & $\mathrm{HP} 4890$ \\
\hline Columna & $\mathrm{SP}-2560$ Capillary Column: $100 \mathrm{~m} \times 0,25 \mathrm{~mm} \times 0,2 \mu \mathrm{m}$ \\
\hline Programación de temperatura & $140^{\circ} \mathrm{C}-5 \mathrm{~min}$, de 140 a $240^{\circ} \mathrm{C}$ a $4{ }^{\circ} \mathrm{C} / \mathrm{min}, 240^{\circ} \mathrm{C}-15 \mathrm{~min}$ \\
\hline Gas carrier & $\mathrm{H}_{2}, 18 \mathrm{~cm} / \mathrm{s}$ \\
\hline Temperatura del inyector & $175^{\circ} \mathrm{C}$ \\
\hline Relación split & $1 / 100$ \\
\hline Volumen de inyección & $1 \mu \mathrm{L}$ \\
\hline Detector & $\mathrm{FID}$ \\
\hline Temperatura del detector & $260^{\circ} \mathrm{C}$ \\
\hline
\end{tabular}




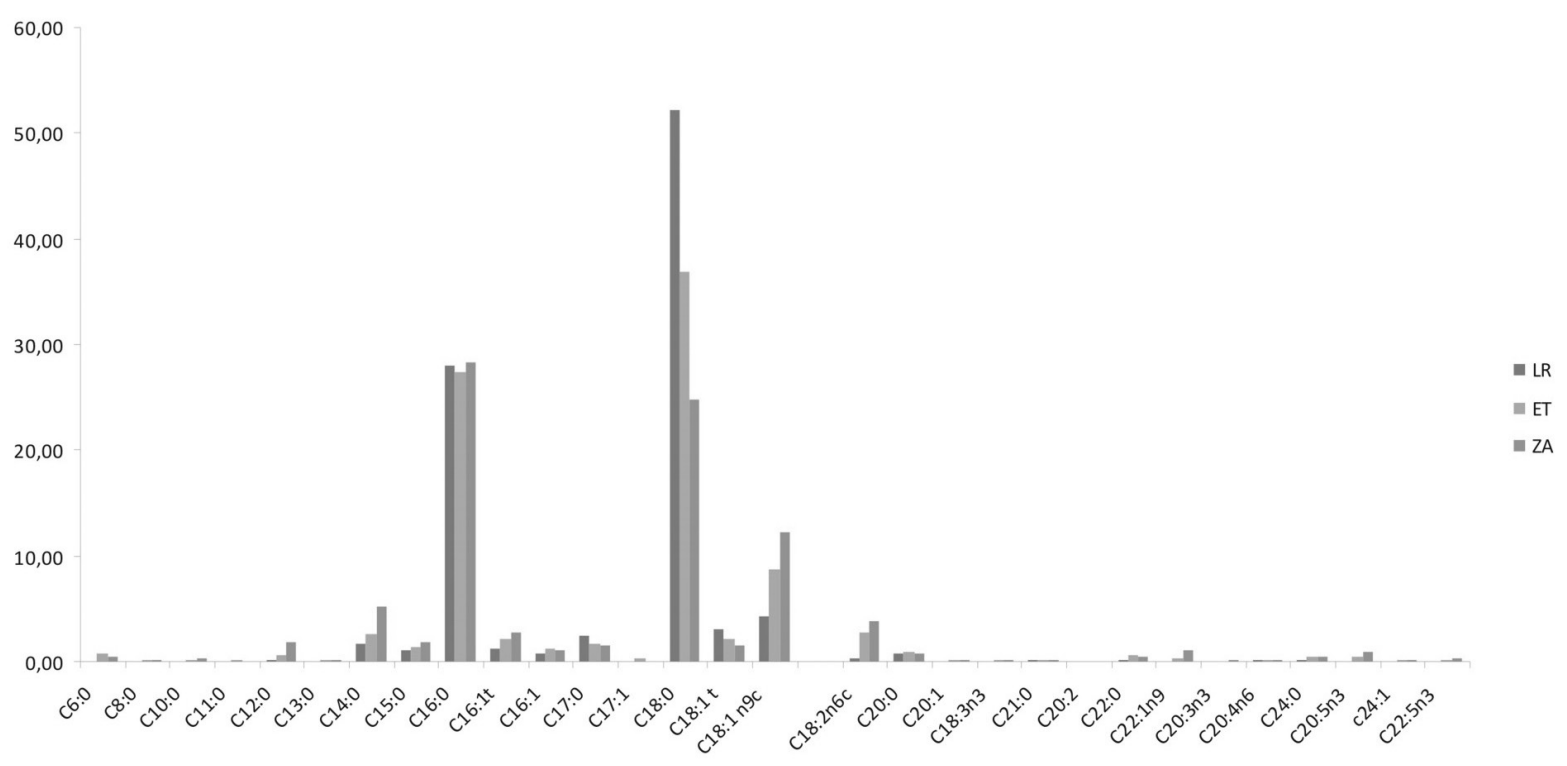

Fig. 3. Gráfico de barras que muestra el porcentaje de cada ácido graso por sitio. LR: Loma Ruiz 1; ET: El Tigre; ZA: Zoko Andi 1.

condiciones y el equipamiento que se detallan en la Tabla 1. El análisis cromatográfico se llevó a cabo por duplicado. La identificación de cada componente se realizó por comparación de los tiempos de retención de cada compuesto con un estándar externo constituido por la mezcla de 37 componentes (Supelco FAME Mix 37).

\section{RESULTADOS}

El principal resultado, común a todos los sitios, es que en todos los tiestos analizados se detectaron ácidos grasos (Fig. 3). Incluso fue posible determinar la presencia de aquellos que en general se degradan rápidamente, como es el caso de los ácidos grasos poliinsturados muy frecuentes en las grasas de los peces (i.e., C20:5n3, C20:4n6 y C20:1; ver Fig. 3). En la Fig. 3 se observan los valores promediados de los ácidos grasos presentes en las muestras de cada sitio. En el caso del sitio Loma Ruíz 1, no fue considerada la muestra 3 (LR3) para realizar este cálculo, mientras que en el sitio El Tigre no se incluyó la muestra 4 (ET4) debido a que, en ambos casos un alto porcentaje de ácidos no pudo ser identificado $y$, en consecuencia, sus valores no son representativos (ver Tablas 2 y 5). Los resultados obtenidos mostraron una preservación diferencial de los ácidos grasos en los tres sitios.
En las muestras del sitio Loma Ruíz 1 se recuperó una importante cantidad de lípidos mientras que en los tiestos cerámicos de los sitios El Tigre y Zoko Andi 1 la presencia de material lipídico fue menor. En consecuencia, los porcentajes en los que se encuentran representados los distintos ácidos en los sitios estarían influidos por cuestiones de preservación. Por lo tanto, con la información derivada de estos estudios solo se pueden plantear tendencias generales. A pesar de esto, es importante destacar que se observa un patrón que indica una frecuencia mayor de ciertos ácidos que se repite en todos los sitios (Fig. 3).

\section{Loma Ruíz 1:}

En todas las piezas analizadas $(\mathrm{N}=6)$ se encontró una considerable cantidad de lípidos (Tabla 2). Los resultados obtenidos de los análisis de ácidos grasos estarían indicando la cocción de carne de grandes herbívoros. En este sentido, los valores de ácido esteárico (C18:0) superan el $30 \%$ en todas las muestras. Asimismo, los valores de ácido palmítico (C16:0) también se aproximan a este porcentaje (Fig. 3). Malainey et al. (1999a) señalan que en los residuos que contienen carne de grandes herbívoros los ácidos esteárico y palmítico representan el 30-40\% de todos los ácidos grasos. Además, la presencia de 
Tabla 2. Composición de ácidos grasos de las muestras provenientes de Loma Ruíz $1 . \operatorname{Tr}=$ trazas valores $<0,02 \%$, ni= no identificados.

\begin{tabular}{|c|c|c|c|c|c|c|}
\hline Ácidos grasos & LR1 & LR2 & LR3 & LR4 & LR5 & LR6 \\
\hline C6:0 & $\operatorname{tr}$ & $\operatorname{tr}$ & 0,70 & 0,02 & $\operatorname{tr}$ & 0,53 \\
\hline $\mathrm{C} 8: 0$ & $\operatorname{tr}$ & $\operatorname{tr}$ & 0,09 & $\operatorname{tr}$ & $\operatorname{tr}$ & \\
\hline C10:0 & 0,03 & 0,03 & 0,38 & 0,02 & 0,05 & \\
\hline C11:0 & $\operatorname{tr}$ & $\operatorname{tr}$ & 0,11 & $\operatorname{tr}$ & $\operatorname{tr}$ & \\
\hline $\mathrm{C} 12: 0$ & 0,14 & 0,13 & 1,24 & 0,09 & 0,12 & 0,73 \\
\hline C13:0 & 0,07 & 0,07 & 0,24 & 0,04 & 0,05 & \\
\hline C14:0 & 1,82 & 2,06 & 4,50 & 1,29 & 1,37 & 2,82 \\
\hline C15:0 & 1,19 & 1,31 & 2,00 & 1,09 & 0,97 & 1,69 \\
\hline $\mathrm{C} 16: 0$ & 28,15 & 29,02 & 26,05 & 29,57 & 25,57 & 29,58 \\
\hline $\mathrm{C} 16: 1 \mathrm{t}$ & 1,24 & 1,37 & 4,57 & 1,20 & 1,06 & 3,40 \\
\hline C16:1 & 0,82 & 1,01 & 1,20 & 0,81 & 0,64 & 1,05 \\
\hline C17:0 & 2,32 & 2,29 & 1,75 & 2,59 & 2,49 & 1,92 \\
\hline $\mathrm{C} 17: 1$ & $\operatorname{tr}$ & $\operatorname{tr}$ & & $\operatorname{tr}$ & $\operatorname{tr}$ & \\
\hline $\mathrm{C} 18: 0$ & 50,75 & 48,55 & 33,39 & 52,71 & 56,90 & 37,19 \\
\hline $\mathrm{C} 18: 1 \mathrm{t}$ & 3,56 & 3,67 & 1,22 & 2,71 & 2,43 & 0,91 \\
\hline $\mathrm{C} 18: 1 \mathrm{n} 9 \mathrm{c}$ & 5,10 & 5,66 & 7,33 & 3,28 & 3,25 & 10,24 \\
\hline $\mathrm{C} 18: 2 \mathrm{n} 6 \mathrm{c}$ & 0,23 & 0,28 & 2,27 & 0,26 & 0,21 & 3,53 \\
\hline $\mathrm{C} 18: 2 \mathrm{t}$ & & & & & & 0,61 \\
\hline C20:0 & 0,80 & 0,76 & 0,99 & 0,76 & 0,87 & 1,21 \\
\hline C20:1 & $\operatorname{tr}$ & 0,02 & 0,05 & $\operatorname{tr}$ & $\operatorname{tr}$ & 0,25 \\
\hline C18:3n3 & $\operatorname{tr}$ & 0,03 & 0,14 & 0,02 & 0,02 & 0,18 \\
\hline $\mathrm{C} 21: 0$ & 0,13 & 0,12 & 0,19 & 0,12 & 0,14 & \\
\hline C20:2 & & & & 0,07 & & \\
\hline $\mathrm{C} 22: 0$ & 0,20 & 0,18 & 0,57 & 0,18 & 0,21 & 0,66 \\
\hline $\mathrm{C} 22: \ln 9$ & & 0,02 & 0,63 & $\operatorname{tr}$ & 0,03 & \\
\hline $\mathrm{C} 20: 3 \mathrm{n} 3$ & & & & $\operatorname{tr}$ & $\operatorname{tr}$ & 0,21 \\
\hline $\mathrm{C} 20: 4 \mathrm{n} 6$ & 0,09 & 0,08 & 0,10 & 0,09 & 0,10 & 0,15 \\
\hline $\mathrm{C} 24: 0$ & 0,11 & 0,11 & 0,48 & 0,12 & 0,12 & \\
\hline $\mathrm{C} 20: 5 \mathrm{n} 3$ & 0,02 & $\operatorname{tr}$ & 0,39 & 0,04 & 0,032 & \\
\hline $\mathrm{C} 22: 5 \mathrm{n} 3$ & & $\operatorname{tr}$ & $\operatorname{tr}$ & $\operatorname{tr}$ & $\operatorname{tr}$ & \\
\hline sum & 96,75 & 96,78 & 89,88 & 97,07 & 96,64 & 96,86 \\
\hline ni & 3,25 & 3,22 & 10,12 & 2,93 & 3,36 & 2,84 \\
\hline
\end{tabular}


Tabla 3. Resultados de la aplicación de los criterios propuestos por Malainey et al. (1999a) en las muestras analizadas.

\begin{tabular}{|c|c|c|c|}
\hline Muestras & $\mathrm{C} 12: 0+\mathrm{C} 14: 0+\mathrm{C} 15: 0$ & $\mathrm{C} 18: 0$ & $\mathrm{C} 18: 1$ \\
\hline \multicolumn{4}{|c|}{ Loma Ruíz 1} \\
\hline LR1 & 3,14 & 50,75 & 8,65 \\
\hline LR2 & 3,49 & 48,55 & 9,33 \\
\hline LR3 & 7,74 & 33,39 & 8,55 \\
\hline LR4 & 2,47 & 52,71 & 6,00 \\
\hline LR5 & 2,46 & 56,90 & 5,68 \\
\hline LR6 & 5,24 & 37,19 & 11,15 \\
\hline \multicolumn{4}{|c|}{ El Tigre } \\
\hline ET1 & 4,2 & 41,96 & 10,61 \\
\hline ET2 & 3,94 & 40,27 & 12,81 \\
\hline ET3 & 4,73 & 26,02 & 12,93 \\
\hline ET4 & 6,01 & 8,76 & 3,84 \\
\hline ET5 & 4,83 & 39,56 & 6,82 \\
\hline ET6 & 5,02 & 29,87 & 11,74 \\
\hline \multicolumn{4}{|c|}{ Zoko Andi 1} \\
\hline ZA1 & 11,05 & 14,03 & 12,92 \\
\hline ZA2 & 4,28 & 46,75 & 7,1 \\
\hline ZA3 & 10,7 & 22,21 & 12,14 \\
\hline ZA4 & 6,2 & 18,96 & 22,08 \\
\hline ZA5 & 11,84 & 22,40 & 12,86 \\
\hline
\end{tabular}

los ácidos behénico (C22:0) y lignocérico (C24:0) es indicativa de grasa animal, como también lo es el mayor porcentaje de ácido oleico (C18:1n9) en relación con el linoleico (C18:2n6; Fig. 3). Asimismo, Malainey et al. (1999a) indican que altos valores de ácido esteárico (27,5\% o más) junto con valores de ácido oleico (C18:1) inferiores al $15 \%$ se relacionarían con la preparación de carne de grandes herbívoros sola o acompañada con la presencia de vegetales. Estas proporciones de ácidos son visibles en la muestra bajo estudio. En consecuencia, podría considerarse un posible uso de recipientes para cocción de herbívoros, quizás acompañados por vegetales. No obstante, estos autores también señalan que los residuos provenientes de la cocción de carne de herbívoros con plantas no son significativamente diferentes de los obtenidos de la preparación de carne y médula, por lo cual no podría descartarse esta segunda opción.

Por otra parte, la presencia de los ácidos eicosapentaenoico (C20:5n3), araquidónico (C20:4n6) y eicosenoico (C20:1) indicaría la presencia de peces o de algún componente de origen marino. Sin embargo, el porcentaje de los mismos es bajo (Tabla 2; Fig. 3), como era de esperar por la rápida degradación que presentan estos compuestos. En síntesis, los resultados obtenidos en Loma Ruíz 1 sugieren la cocción de grandes herbivoros $y$, posiblemente de plantas y/o de médula ósea junto con la presencia de recursos de origen marino. Estas categorías de recursos son inferibles a partir de la aplicación de los criterios e índices propuestos por Malainey et al. (1999a; Tabla 3) y Eerkens (2005; Tabla 4).

\section{El Tigre:}

En todos los tiestos analizados de este sitio $(\mathrm{N}=6)$ la presencia de material lipídico fue menor (Tabla 5). En principio, los resultados obtenidos sugieren la cocción de grandes herbívoros. En este sentido, los valores de C18:0 (ácido esteárico) y C16:0 (ácido palmítico) en 5 muestras se aproximan o superan el $30 \%$, mientras que en la restante no exceden el $11 \%$. Sin embargo, en este caso los valores de ácido esteárico y palmítico presentan los porcentajes más altos en comparación al resto de los ácidos detectados. Además, la presencia de los ácidos behénico y lignocérico así como el mayor porcentaje de ácido oleico en comparación con el linoleico indicaría la presencia de grasa animal (Fig. 3). La propuesta de cocción predominante de grandes herbivoros se ve reforzada también por los bajos valores de ácido oleico y de ácidos grasos de cadena media (láurico: C12:0, mirístico: C14:0 y pentadecanoico: C15:0), que sugieren una reducida proporción en la cocción de vegetales (Fig. 3; Malainey et al. 1999a). Finalmente, se detectaron algunos ácidos (i.e., eicosenoico: C20:1 y araquidónico: C20:4n6) que se corresponderían con la presencia de peces o de productos marinos (i.e., algas, moluscos, etc.). Estos resultados se condicen con aquellos derivados de los criterios e índices propuestos por Malainey et al. (1999a; Tabla 3) y Eerkens (2005; Tabla 4), por lo cual 
Tabla 4. Resultados del cálculo de los índices desarrollados por Eerkens (2005) en las muestras analizadas.

\begin{tabular}{|c|c|c|c|c|}
\hline \multirow{2}{*}{ Muestras } & \multicolumn{4}{|c|}{ Índices } \\
\hline & $\mathrm{C} 15: 0+\mathrm{C} 17: 0 / \mathrm{C} 18: 0$ & $\mathrm{C} 16.1 / \mathrm{C} 18: 1$ & $\mathrm{C} 16: 0 / \mathrm{C} 18: 0$ & $\mathrm{C} 12: 0 / \mathrm{C} 14: 0$ \\
\hline \multicolumn{5}{|c|}{ Loma Ruíz 1} \\
\hline LR1 & 0,06 & 0,23 & 0,55 & 0,07 \\
\hline LR2 & 0,07 & 0,25 & 0,59 & 0,06 \\
\hline LR3 & 0,11 & 0,67 & 0,78 & 0,27 \\
\hline LR4 & 0,06 & 0,33 & 0,56 & 0,06 \\
\hline LR5 & 0,06 & 0,29 & 0,44 & 0,08 \\
\hline LR6 & 0,09 & 1,29 & 0,79 & 0,25 \\
\hline \multicolumn{5}{|c|}{ El Tigre } \\
\hline ET1 & 0,07 & 0,25 & 0,64 & 0,24 \\
\hline ET2 & 0,06 & 0,25 & 0,67 & 0,10 \\
\hline ET3 & 0,09 & 0,30 & 1,19 & 0,31 \\
\hline ET4 & 0,13 & 0,27 & 1,24 & 0,61 \\
\hline ET5 & 0,09 & 0,45 & 0,62 & 0,23 \\
\hline ET6 & 0,09 & 0,35 & 0,97 & 0,25 \\
\hline \multicolumn{5}{|c|}{ Zoko Andi 1} \\
\hline ZA1 & 0,25 & 0,32 & 2,01 & 0,47 \\
\hline ZA2 & 0,08 & 0,37 & 0,62 & 0,11 \\
\hline ZA3 & 0,16 & 0,40 & 1,20 & 0,40 \\
\hline ZA4 & 0,11 & 0,17 & 1,61 & 0,39 \\
\hline ZA5 & 0,17 & 0,29 & 1,17 & 0,27 \\
\hline
\end{tabular}

es posible sugerir que los resultados obtenidos a partir de estas muestras evidencian la cocción de grandes herbívoros, posiblemente acompañados por plantas y la presencia de algún componente marino.

\section{Zoko Andi 1:}

En la muestra analizada en Zoko Andi 1 $(\mathrm{N}=5)$, al igual que en El Tigre, se encontró muy poca cantidad de lípidos (Tabla 6). No obstante, los resultados obtenidos sugieren las mismas tendencias que las propuestas para los restantes sitios. En este sentido, se observa una alta frecuencia de los ácidos esteárico y palmítico que indicaría la cocción de grandes herbívoros (Fig. 3; Buonasera, 2005; Malainey et al. 1999a). Esta inferencia se ve reforzada por el mayor porcentaje en el que se encuentra el ácido oleico en relación con el linoleico así como la presencia de los ácidos behénico y lignocérico, propios de grasas animales. Asimismo, los valores de ácido esteárico junto con los valores de ácido oleico sugieren también la preparación de carne de grandes herbívoros sola o acompañada por plantas. Sin embargo, como se mencionó anteriormente, estos residuos no son diferentes de aquellos producidos por la preparación de carne y médula, por lo cual no podría realizarse la distinción entre estos recursos (Malainey et al. 1999a). Por último, la presencia aunque en bajo porcentaje de los ácidos eicosapentaenoico (C20:5n3), araquidónico (C20:4n6) y eicosenoico (C20:1) sugiere la presencia de peces o de productos de origen marino (Tabla 6; Fig. 3). Estas tendencias son consistentes con aquellas obtenidas a partir de los criterios e índices desarrollados por Malainey et al. (1999a; Tabla 3) y Eerkens (2005; Tabla 4). En suma, los análisis de ácidos grasos de las muestras de este sitio sugieren la cocción de grandes herbívoros, quizás acompañada por plantas y/o médula y la presencia de recursos de origen marino. 
Tabla 5. Composición de ácidos grasos de las muestras provenientes del sitio El Tigre.

\begin{tabular}{|c|c|c|c|c|c|c|}
\hline Ácidos grasos & ET1 & ET2 & ET3 & ET4 & ET5 & ET6 \\
\hline c6:0 & 0,16 & 0,29 & 1,14 & 9,42 & 1,34 & 0,64 \\
\hline $\mathrm{C} 8: 0$ & $\operatorname{tr}$ & $\operatorname{tr}$ & 0,13 & 0,64 & 0,31 & \\
\hline c10:0 & 0,03 & 0,08 & 0,20 & 0,82 & 0,28 & \\
\hline $\mathrm{c} 11: 0$ & $\operatorname{tr}$ & 0,10 & $\operatorname{tr}$ & 0,58 & $\operatorname{tr}$ & \\
\hline $\mathrm{C} 12: 0$ & 0,58 & 0,27 & 0,84 & 2,01 & 0,60 & 0,72 \\
\hline C13:0 & 0,11 & 0,06 & 0,15 & $\operatorname{tr}$ & 0,48 & 0,22 \\
\hline C14:0 & 2,34 & 2,51 & 2,69 & 3,26 & 2,58 & 2,78 \\
\hline C15:0 & 1,28 & 1,16 & 1,20 & 0,74 & 1,65 & 1,50 \\
\hline C16:0 & 26,86 & 27,00 & 31,19 & 10,91 & 24,77 & 29,24 \\
\hline $\mathrm{C} 16: 1 \mathrm{t}$ & 1,84 & 2,29 & 2,21 & 0,61 & 2,05 & 2,82 \\
\hline $\mathrm{C} 16: 1$ & 0,87 & 1,02 & 1,73 & 0,45 & 1,08 & 1,29 \\
\hline $\mathrm{C} 17: 0$ & 1,76 & 1,48 & 1,30 & 0,44 & 2,00 & 1,23 \\
\hline $\mathrm{C} 17: 1$ & & & & & 0,23 & \\
\hline $\mathrm{C} 18: 0$ & 41,96 & 40,27 & 26,02 & 8,76 & 39,56 & 29,87 \\
\hline $\mathrm{C} 18: 1 \mathrm{n} 9 \mathrm{t}$ & 2,71 & 2,80 & 0,90 & & 1,93 & \\
\hline $\mathrm{C} 18 \mathrm{~s} \ln 9 \mathrm{c}$ & 7,90 & 10,01 & 12,03 & 3,84 & 4,89 & \\
\hline $\mathrm{C} 18: 1 \mathrm{t}$ & & & & & & 2,06 \\
\hline $\mathrm{C} 18 \mathrm{~s} 1 \mathrm{c}$ & & & & & & 11,74 \\
\hline $\mathrm{C} 18: 2 \mathrm{n} 6 \mathrm{t}$ & $\operatorname{tr}$ & $\operatorname{tr}$ & $\operatorname{tr}$ & & & \\
\hline $\mathrm{C} 18: 2 \mathrm{n} 6 \mathrm{c}$ & 2,28 & 2,05 & 5,09 & 1,35 & 1,62 & 3,33 \\
\hline $\mathrm{C} 18: 2 \mathrm{t}$ & & & & & & 0,38 \\
\hline $\mathrm{C} 20: 0$ & 0,89 & 0,93 & 0,74 & 0,31 & 1,23 & 0,99 \\
\hline C20:1 & 0,27 & 0,36 & 0,13 & $\operatorname{tr}$ & 0,07 & 0,31 \\
\hline $\mathrm{C} 18: 3 \mathrm{n} 3$ & 0,08 & 0,06 & 0,12 & $\operatorname{tr}$ & 0,05 & 0,14 \\
\hline $\mathrm{C} 18: 3 \mathrm{t}$ & & & & & & 0,44 \\
\hline $\mathrm{C} 21: 0$ & 0,15 & 0,13 & 0,15 & & 0,14 & 0,12 \\
\hline $\mathrm{C} 20: 2$ & & & & & $\operatorname{tr}$ & 2,14 \\
\hline $\mathrm{C} 22: 0$ & 0,50 & 0,58 & 0,80 & 0,38 & 0,45 & 1,00 \\
\hline $\mathrm{C} 22: 1 \mathrm{n} 9$ & 0,32 & 0,24 & 0,28 & 0,30 & 0,33 & \\
\hline $\mathrm{C} 20: 3 \mathrm{n} 3$ & & & & & $\operatorname{tr}$ & 0,29 \\
\hline C20:3n6 & & & & & & 0,17 \\
\hline $\mathrm{C} 20: 4 \mathrm{n} 6$ & 0,14 & 0,08 & 0,09 & 2,82 & 0,25 & \\
\hline C24:0 & 0,36 & 0,38 & 0,38 & 0,31 & 0,52 & 0,63 \\
\hline $\mathrm{C} 20: 5 \mathrm{n} 3$ & 0,23 & 0,39 & 0,64 & 7,59 & 0,46 & \\
\hline $\mathrm{c} 24: 1$ & 0,08 & 0,15 & & & & 0,27 \\
\hline $\mathrm{C} 22: 5 \mathrm{n} 3$ & 0,07 & 0,18 & 0,22 & 3,99 & 0,15 & \\
\hline sum & 93,60 & 94,78 & 90,40 & 59,53 & 88,79 & 94,32 \\
\hline $\mathrm{ni}$ & 6,41 & 5,22 & 9,60 & 40,47 & 11,21 & 5,41 \\
\hline
\end{tabular}


Tabla 6. Composición de ácidos grasos de las muestras provenientes del sitio Zoko Andi 1 . $\mathrm{Tr}=$ trazas valores $<0,02 \%$, ni= no identificados.

\begin{tabular}{|c|c|c|c|c|c|}
\hline Ácidos grasos & $\mathrm{Z1}$ & $\mathrm{Z2}$ & $\mathrm{Z3}$ & $\mathrm{Z4}$ & Z5 \\
\hline $\mathrm{C} 6: 0$ & 0,42 & 0,05 & 0,82 & 1,00 & 0,11 \\
\hline $\mathrm{C} 8: 0$ & 0,32 & 0,01 & 0,31 & 0,11 & 0,14 \\
\hline $\mathrm{C} 10: 0$ & 0,63 & 0,05 & 0,48 & 0,33 & 0,24 \\
\hline $\mathrm{C} 11: 0$ & $\operatorname{tr}$ & 0,04 & & & \\
\hline $\mathrm{c} 12: 0$ & 2,79 & 0,28 & 2,45 & 1,39 & 2,12 \\
\hline C13:0 & 0,33 & 0,14 & 0,20 & & 0,19 \\
\hline $\mathrm{C} 14: 0$ & 5,83 & 2,38 & 6,07 & 3,55 & 7,85 \\
\hline C15:0 & 2,43 & 1,62 & 2,18 & 1,26 & 1,87 \\
\hline C16:0 & 28,26 & 29,43 & 26,73 & 30,54 & 26,43 \\
\hline $\mathrm{C} 16: 1 \mathrm{t}$ & 3,31 & 1,65 & 3,77 & 2,50 & 2,72 \\
\hline $\mathrm{C} 16: 1$ & 0,94 & 1,03 & 1,18 & 1,36 & 1,05 \\
\hline $\mathrm{C} 17: 0$ & 1,09 & 2,22 & 1,47 & 0,90 & 2,14 \\
\hline $\mathrm{C} 17: 1$ & & $\operatorname{tr}$ & $\operatorname{tr}$ & & \\
\hline $\mathrm{C} 18: 0$ & 14,03 & 46,75 & 22,21 & 18,96 & 22,40 \\
\hline $\mathrm{C} 18: 1 \mathrm{t}$ & & 2,80 & 1,42 & 0,84 & 1,05 \\
\hline $\mathrm{C} 18: 1 \mathrm{n} 9 \mathrm{c}$ & 12,92 & 4,30 & 10,72 & 21,24 & 11,81 \\
\hline $\mathrm{C} 18: 2 \mathrm{n} 6 \mathrm{t}$ & & $\operatorname{tr}$ & tr & tr & $\operatorname{tr}$ \\
\hline $\mathrm{C} 18: 2 \mathrm{n} 6 \mathrm{c}$ & 5,00 & 0,78 & 3,56 & 4,34 & 5,29 \\
\hline C20:0 & 0,78 & 0,83 & 0,67 & 1,06 & 0,80 \\
\hline $\mathrm{C} 20: 1$ & 0,19 & 0,05 & 0,09 & 0,24 & 0,15 \\
\hline $\mathrm{C} 18: 3 \mathrm{n} 3$ & 0,18 & 0,05 & 0,15 & 0,10 & 0,15 \\
\hline $\mathrm{C} 21: 0$ & & 0,15 & 0,14 & 0,08 & 0,12 \\
\hline $\mathrm{C} 20: 2$ & & $\operatorname{tr}$ & & & $\operatorname{tr}$ \\
\hline $\mathrm{C} 22: 0$ & 0,57 & 0,30 & 0,41 & 0,59 & 0,59 \\
\hline $\mathrm{C} 22: \ln 9$ & 0,45 & 0,13 & 0,26 & 0,56 & 3,97 \\
\hline $\mathrm{C} 20: 3 \mathrm{n} 3$ & & $\operatorname{tr}$ & $\operatorname{tr}$ & 0,13 & $\operatorname{tr}$ \\
\hline $\mathrm{C} 20: 4 \mathrm{n} 6$ & 0,25 & 0,11 & 0,28 & 0,16 & 0,11 \\
\hline $\mathrm{C} 24: 0$ & 0,68 & 0,23 & 0,31 & 0,50 & 0,50 \\
\hline $\mathrm{C} 20: 5 \mathrm{n} 3$ & 1,16 & 0,07 & 1,15 & 0,67 & 1,89 \\
\hline C24:1 & & tr & tr & tr & 0,14 \\
\hline $\mathrm{C} 22: 5 \mathrm{n} 3$ & & 0,04 & 0,49 & 0,27 & 0,18 \\
\hline sum & 82,55 & 95,49 & 87,47 & 92,59 & 93,99 \\
\hline ni & 17,45 & 4,51 & 12,53 & 7,41 & 6,01 \\
\hline
\end{tabular}




\section{DISCUSIÓN Y CONCLUSIONES}

Los resultados obtenidos de los análisis de ácidos grasos de las 17 muestras de tiestos cerámicos provenientes de los tres sitios, permitieron identificar categorías amplias de recursos. En este sentido, los estudios realizados en los tres sitios sugieren la cocción de grandes herbivoros, posiblemente acompañados por plantas y/o médula ósea y la presencia de un componente de fauna marina, muy probablemente peces. Esta información es concordante y refuerza algunos de los resultados derivados de los análisis zooarqueológicos e isotópicos mencionados en la introducción de este trabajo. En este sentido, se destaca el aporte de estos nuevos datos a la comprensión del rol de los peces marinos en la subsistencia. Como ya fuera discutido en otros trabajos (Alcaráz, 2012; Martínez et al. 2009a; Stoessel, 2012; Stoessel \& Martínez, 2014) el registro zooarqueológico de peces durante el Holoceno tardío inicial es prácticamente inexistente. Sin embargo, desde otras líneas de evidencia como los isótopos estables de restos óseos humanos ya fue planteado el consumo de peces marinos para este lapso (Martínez et al. 2009a). En consecuencia, el registro de ácidos grasos vinculados a la explotación de estos recursos en el sitio Loma Ruíz 1, para el período en cuestión es otra línea de evidencia independiente concordante con este planteo.

Por otro lado, la recuperación de ácidos grasos de peces marinos en este sitio tiene otras implicaciones. En este sentido, los estudios realizados en distintos sitios del área mostraron que hubo una explotación diferencial de peces a través del paisaje que respondería a la localización particular de los recursos y a la funcionalidad de los sitios (Alcaráz, 2012; Martínez et al. 2009b; Stoessel, 2012). En este caso, la explotación sistemática de peces marinos se registra en los sitios ubicados en la franja que va entre los ca. 15-4 km de la costa atlántica. Por ejemplo, en el sitio La Primavera, datado en el Holoceno tardío Inicial (ca. 3.000-2.800 años AP) y ubicado a ca. $20 \mathrm{~km}$ de la costa, los valores de isótopos estables en restos óseos humanos indican un componente acuático, marino, en las dietas. Sin embargo, en esta base residencial de actividades múltiples se recuperaron escasos especimenes de peces (NISP=3; Stoessel, 2012). Dadas estas evidencias se planteó que la escasa presencia de restos de peces indicaría que el consumo se llevó a cabo en otro sector del espacio. En este sentido, los grupos habrían ocupado los sectores costeros, explotando in situ recursos de este sector del paisaje, como parte de su ciclo anual de actividades. Esto se vio reforzado por las investigaciones en la localidad arqueológica San Antonio, localizada a ca. $4 \mathrm{~km}$ del mar, con una cronología de ca. 1.000-800 años AP. En estos sitios, caracterizados como bases residenciales de actividades múltiples, los restos de peces marinos (bagre de mar, corvina rubia, chuchos, condrictios y sciénidos indeterminados) dominan ampliamente los conjuntos faunísticos (i.e., NISP total: 3.134) y poseen evidencias de consumo. Las evidencias de estos contextos (i.e., especies marinas eurihalinas) llevaron a proponer la ocupación estacional de estos sectores del paisaje, donde se habrían llevado a cabo principalmente actividades de subsistencia, relacionadas a la obtención de peces (Martínez et al. 2010).

Dado este patrón temporal y espacial descripto para la obtención y consumo de peces, cabe preguntarse qué indican los ácidos grasos recuperados en Loma Ruíz 1. Este sitio está ubicado en un área de dunas y pequeñas lagunas, salobres y dulces, en el interior de cordones eólicos que se ubican a ca. $30 \mathrm{~km}$ de la costa Atlántica. Como se destacó anteriormente, en este sitio no se recuperaron restos óseos de peces. Sin embargo, la composición de la muestra de ácidos grasos proveniente de este sitio indica la presencia de un componente marino que, dado el registro zooarqueológico conocido para el área y dados los criterios experimentales de identificación de clases de fauna mediante señales de ácidos grasos, muy probablemente se corresponda con peces. La presencia de ácidos grasos de peces en los tiestos de Loma Ruíz 1 sugeriría que como parte del sistema de movilidad, con un alto componente residencial (Martínez, 2008-2009), los contenedores habrían sido trasladados a través del paisaje. Es decir, los peces habrían sido almacenados $y / 0$ cocinados en otro lugar (sector costero) y las vasijas luego trasladadas hacia el interior. En este sentido, la presencia de estos contenedores cerámicos en 
lugares donde los peces no han sido explotados implica la utilización de los mismos en otros contextos espaciales y situacionales.

Respecto del modelo de subsistencia descripto más arriba estos estudios preliminares de ácidos grasos apoyaron las tendencias generales ya conocidas sobre la subsistencia para el Holoceno tardío en general, pero no brindaron información de mayor detalle que permita discutir la existencia de procesos de cambio en la explotación de recursos (i.e., diversificación, intensificación, etc.) propuestos hacia el Holoceno tardío Final. Estos resultados podrían estar mediados por la preservación diferencial de los ácidos recuperados de las vasijas de estos sitios. En este sentido, el sitio que representa al Holoceno tardío Inicial (Loma Ruíz 1), posee una mayor preservación de ácidos que aquellos sitios (El Tigre y Zoko Andi 1) cronológicamente vinculados al Holoceno tardío Final, momento en que se proponen los principales cambios en la subsistencia.

\section{AGRADECIMIENTOS}

Este trabajo fue realizado gracias a subsidios provistos por el CONICET (PIP-CONICET $\mathrm{N}^{\circ}$ 338/10) y ANPCyT (PICT $N^{\circ}$ 264/2006). Al INCUAPA-CONICET y a la FACSO-UNCPBA por brindar soporte institucional $e$ instalaciones. Los autores agradecen a la Dra. Danae Fiore por la lectura del manuscrito y sus valiosos comentarios y sugerencias.

\section{BIBLIOGRAFÍA}

Alcaráz, A. P. (2012). Análisis zooarqueológico y tafonómico de restos óseos de pequeños vertebrados del curso inferior del río Colorado (pcia. de Buenos Aires) durante el Holoceno tardío. Aportes para el conocimiento de la subsistencia y procesos de formación. Tesis de Licenciatura inédita. Facultad de Ciencias Sociales de Olavarría. Universidad Nacional del Centro de la Provincia de Buenos Aires. Olavarría.

AOCS, Official Methods and Recommended Practices of American Oil Chemist Society (5 $5^{\text {th }}$ ed). (1997). Champaign, Illinois: American Oil Chemists Society.

Armentano, G. (2012). Arqueología del curso inferior del Río Colorado. Estudio tecnológico de las colecciones líticas de Norpatagonia Oriental durante el Holoceno tardío.
Departamentos de Villarino y Patagones, Provincia de Buenos Aires, Argentina. Tesis Doctoral Inédita. Facultad de Ciencias Sociales de Olavarría. Universidad Nacional del Centro de la Provincia de Buenos Aires. Olavarría. En co-tutela internacional con Université de Paris Ouest Nanterre-la défense. France arts, Lettres et Sciences Humaines. École doctoral: Milieux, cultures et sociétés du passé et du présent.

Armentano, G., Martínez, G. \& Landini, C. (2013). El sitio Loma Ruíz 1: Estrategias tecnológicas líticas durante el Holoceno tardío inicial en la transición pampeano-patagónica oriental. Revista del Museo de Antropología, 6, 21-40.

Babot, M. P., Mazzia, N. \& Bayón, C. (2007). Procesamiento de recursos en la región pampeana bonaerense: aportes del instrumental de molienda de las localidades arqueológicas El Guanaco y Cerro La China. En C. Bayón, A. Pupio, M.I. González, N. Flegenheimer \& M. Frère (Eds.), Arqueología en las Pampas (pp. 635-657). Buenos Aires: Sociedad Argentina de Antropología

Bonomo, M., Colobig, M., \& Mazzia, N. (2012). Análisis de residuos orgánicos y microfósiles silíceos de la "cuchara" de cerámica del sitio arqueológico Cerro Tapera Vázquez (Parque Nacional Pre-Delta, Argentina). $R$. Museu Arq. Etn., 22, 31-50.

Buonasera, T. (2005). Fatty acid analysis of prehistoric burned rocks: a case study from central California. Journal of Archaeological Science, 32, 957-965.

Charters, S., Evershed, R. P., Blinkhorn, P. W., \& Denham, V. (1995). Evidence for the mixing of fats and waxes in archaeological ceramics. Archaometry, 37(1), 113 127.

Costa Angrisani, R. \& Constenla., D. (2010). Sobre yapepós, ñaembés y cambuchís: aproximaciones a la funcionalidad de vasijas cerámicas a partir de la determinación de ácidos grasos residuales en tiestos recuperados en contextos arqueológicos en el sur de Brasil. En M. Berón, L. Luna, M. Bonomo, C. Montalvo, C. Aranda \& M. Carrera Aizpitarte (Eds.), Mamül Mapu: pasado y presente desde la arqueología pampeana (pp. 5-52). Buenos Aires: Editorial Libros del Espinillo.

Di Prado, V., Scalise, R., Poiré, D. G., Canalicchio, J. M. \& Gómez Peral, L. (2007). Análisis de elementos colorantes provenientes del sitio Calera (Sierras Bayas, región pampeana). Una exploración del uso social y ritual de los pigmentos. En C. Bayón, A. Pupio, M.I. González, N. Flegenheimer \& M. Frére (Eds.), Arqueología en las Pampas (pp. 765-780). Buenos 
Aires: Sociedad Argentina de Antropología.

Eerkens, J. W. (2005). GC-MS analysis and fatty acid ratios of archaeological potsherds from the western great basin of North America. Archaeometry, 47(1), 83-102.

Eerkens, J. W. 2007. Organic residue analysis and the decomposition of fatty acids in ancient potsherds. En $\mathrm{H}$. Barnard \& J.W. Eerkens (Eds.), Theory and Practice in Archaeological residue analysis (pp. 90-98). Oxford: BAR, International Series. Archaeopress.

Evershed, R. P., Heron, C., Charters, S., \& Goad, L. J. (1992). Chemical Analysis of Organic Residues in Ancient Pottery: Methodological Guidelines and Applications. En R. White \& H. Page (Eds.), Organic Residues in Archaeology: Their Identification and Analysis (pp. 11-25). London: United Kingdom Institute for Conservation.

Evershed, R. P., Mottram, H. R., Dudd, S. N., Charters, S., Stott, A. W., Lawrence, G. J.,... Reeves, V. (1997). New criteria for the identification of animal fats preserved in archaeological pottery. Naturwissenschaften, 84, 402-406.

Evershed, R. P., Dudd, S. N., Charters, S., Mottram, H., Stott, A. W., Raven, A.,... Bland, H. A. (1999). Lipids as carriers of anthropogenic signals from prehistory. Phil. Trans. R. Soc. Lond. B, 354, 19-31.

Evershed, R. P., Dudd, S. N., Copley, M. S., Berstan, R., Stott, A. W., Mottram, H.,...Crossman, Z. (2002). Chemistry of archaeological animal fats. Accounts of Chemical Research, 35(8), 660-668.

Evershed, R. P., Copley, M.S., Dickson, L. \& Hansel, F.A. (2008). Experimental evidence for the processing of marine animal products and other commodities containing polyunsaturated fatty acids in pottery vessels. Archaeometry, 50(1), 101-113.

Fiore, D., Maier, M., Parera, S. D., Orquera, L., \& Piana, E. (2008). Chemical analyses of the earliest pigment residues from the uttermost part of the planet (Beagle Channel region, Tierra del Fuego, Southern South America). Journal of Archaeological Science, 35, 3047-3056.

Folch, J., Lees, M. \& Sloane-Stanley, G. H. (1957). A simple method for isolation and purification of total lipids from animal tissues. J. Biological Chemistry, 226, $497-$ 507.

Frère, M. M., Constenla, D., Bayón, C., \& González, M. I. (2010). Estudios actualísticos sobre recursos silvestres mediante en empleo de análisis químico. En M. Berón, L. Luna, M. Bonomo, C. Montalvo, C. Aranda \& M. Carrera Aizpitarte (Eds.), Mamül Mapu: pasado y presente desde la arqueología pampeana (pp. 65-76). Buenos Aires: Editorial Libros del Espinillo.

Gómez Otero, J., Constenla, D., \& Schuster, V. (2014). Análisis de isótopos estables de carbono y nitrógeno y cromatografía gaseosa en cerámica arqueológica del nordeste de la provincia de Chubut (Patagonia Argentina). Arqueología, 20(2), 263-284.

González, M. I. (2005). Arqueología de alfareros, cazadores $y$ pescadores pampeanos. Buenos Aires: Sociedad Argentina de Antropología.

González de Bonaveri, M. I. \& Frère, M. M. (2002). Explorando algunos usos prehispánicos de la alfarería pampeana. En D.L. Mazzanti, M.A. Berón \& F.W. Oliva (Eds.), Del Mar a los Salitrales. Diez mil Años de Historia Pampeana en el Umbral del Tercer Milenio (pp. 31-40). Mar del Plata: Laboratorio de Arqueología, Facultad de Humanidades, Universidad Nacional de Mar del Plata.

Illescas, F., Cañizo, A., Musaubach, M. G. \& Berón, M. A. (2012). De ollas, aceites y otras yerbas. Análisis complementarios sobre alfarería pampeana. En M. P. Babot, M. Marschoff \& F. Pazzarelli (Eds.), Las manos en la masa. Arqueologías, Antropologías e Historias de la Alimentación en Suramérica (pp. 387-404). Museo de Antropología, Instituto de Arqueología de Córdoba.

Isaksson, S. (2009). A long-term perspective on prehistoric pottery use in southern and eastern middle Sweden based on lipid residue analyses. Current Swedish Archaeology, 17, 131-149.

Koirala, B. \& Rosentreter, J. (2009). Examination of prehistoric artifacts via fatty acid methyl ester (FAME) techniques using modern environmental stewardship. Journal of Archaeological Science, 36, 1229-1242.

Maier, M. S., De Faria, D. L. A., Boschín, M. T., Parera, S. D. \& Del Castillo Bernal, M. F. (2007). Combined use of vibrational spectroscopy and GC-MS methods in the characterization of archaeological pastes from Patagonia. Vibrational Spectroscopy, 44, 182-186.

Malainey, M. E. (2007). Fatty acid analysis of archaeological residues: procedures and possibilities. En H. Barnard \& J.W. Eerkens (Eds.), Theory and Practice in Archaeological residue analysis (pp. 77-89). Oxford: BAR, International Series. Archaeopress.

Malainey, M. E., Przybylski, R. \& Sherriff, B. L. (1999a). The effects of thermal and oxidative degradation on the fatty acid composition of food plants and animals of western Canada: Implications for the identification of archaeological vessel residues. Journal of 
Archaeological Science, 26, 93-103.

Malainey, M. E., Przybylski, R., \& Sherriff, B. L. (1999b). The fatty acid composition of native food plants and animals of western Canada. Journal of Archaeological Science, 26, 83-94.

Malainey, M. E., Przybylski, R., \& Sherriff, B. L. (1999c). Identifying the former contents of Late Precontact Period pottery vessels from western Canada using gas chromatography. Journal of Archaeological Science, 26, 425-438.

Martínez, G. (2008-2009). Arqueología del curso inferior del río Colorado: estado actual del conocimiento e implicaciones para la dinámica poblacional de cazadores recolectores pampeano-patagónicos. CazadoresRecolectores del Cono Sur. Revista de Arqueología, 3, 71-92.

Martínez, G., Zangrando, A. F., \& Prates, L. (2009a). Isotopic ecology and human paleodiets in the lower basin of the Colorado River (Buenos Aires Province, Argentina). International Journal of Osteoarchaeology, 281-296.

Martínez, G., Stoessel, L. \& Armentano, G. (2009b). Cronología, procesos de formación y ocupaciones humanas en el sitio El Tigre (pdo. de Patagones, pcia. de Buenos Aires). Relaciones de la Sociedad Argentina de Antropología, XXXIV, 177-199.

Martínez, G., Armentano, G., Stoessel, L., Martínez, G. A., Alcaráz, A. P., González, N., \& Santos, F. (2010). Resultados Preliminares de la localidad arqueológica San Antonio (curso inferior del río Colorado Pdo. Villarino, pcia. de Buenos Aires). En M. Berón, L. Luna, M. Bonomo, C. Montalvo, C. Aranda \& M. Carrera Aizpitarte (Eds.), Mamül Mapu: pasado y presente desde la arqueología pampeana (pp. 8598). Ayacucho, Buenos Aires: Editorial Libros del Espinillo.

Martínez, G., Madrid, P., \& Bonetti, A. (2011). Análisis tecnomorfológico de la alfarería del sitio Loma Ruíz 1 (curso inferior del río Colorado, provincia de Buenos Aires). Trabajo presentado en el VI Congreso de Arqueología de la Región Pampeana, la Plata.

Martínez, G., Martínez, G. A., Stoessel, L., Alcaráz, A. P., Santos Valero, F., Flensborg, G., Bayala, P., \& Onorato, J. F. (2014). Resultados preliminares del sitio arqueológico Zoko Andi 1. Aportes para la arqueología del curso inferior del río Colorado (provincia de Buenos Aires). Revista del Museo de Antropología, 7(1), 105-114.

Mazzia, N. (2010). Lugares y paisajes de cazadores recolectores en la pampa bonaerense: cambios y continuidades durante el Pleistoceno final-Holoceno. Tesis Doctoral
Inédita. Facultad de Ciencias Naturales y Museo. Universidad Nacional de La Plata. La Plata.

Mazzia, N. \& Weitzel, C. (2008). De usos y recursos. Análisis funcional y de sustancias adheridas en artefactos líticos tallados. Libro de resúmenes del $V$ Congreso de Arqueología de la Región Pampeana Argentina, pp. 40. Santa Rosa, La Pampa.

Morrison, W. R. \& Smith, L.M. (1964). Preparation of fatty acid methyl esters and dimethylacetals from lipids boron fluoride-methanol. Lipid Research, 5, 600-608.

Pérez, M., Acosta, I., Naranjo, G. \& Malec, L. (2013). Uso de la alfarería y conductas alimenticias en el humedal del Paraná inferior a través del análisis de ácidos grasos. Cuadernos del Instituto Nacional de Antropología y Pensamiento Latinoamericano. Series Especiales, 1(1), 26-45.

Pérez Meroni, M., Paleo, M. C., Pochettino, M. L., \& Lema, V. S. (2010). Procesamiento y consumo de vegetales por grupos cazadores-recolectores del Holoceno tardío, en los partidos de Magdalena y Punta Indio, provincia de Buenos Aires. En M. Berón, L. Luna, M. Bonomo, C. Montalvo, C. Aranda y M. Carrera Aizpitarte (Eds.), Mamül Mapu: pasado y presente desde la arqueología pampeana (pp. 87-102). Buenos Aires: Editorial Libros del Espinillo.

Schuster, V. (2012). Organización tecnológica de la cerámica de grupos cazadores-recolectores de la costa centroseptentrional de Patagonia (provincia del Chubut, Argentina). Tesis Doctoral Inédita. Facultad de Ciencias Sociales de Olavarría. Universidad Nacional del Centro de la Provincia de Buenos Aires. Olavarría.

Skibo, J. M. (1992). Pottery Function. A Use-alteration Perspective. New York and London: Plenum Press.

Spangenberg, J. E., Jacomet, S., \& Schibler, J. (2006). Chemical analyses of organic residues in archaeological pottery from Arbon Bleiche 3, Switzerland- evidence for dairying in the late Neolithic. Journal of Archaeological Science, 33, 1-13.

Stoessel, L. (2012). Análisis zooarqueológicos en el curso inferior del río Colorado (provincia de Buenos Aires). Aportes para la subsistencia de cazadores-recolectores en el Holoceno tardío. Tesis Doctoral Inédita. Facultad de Ciencias Sociales de Olavarría. Universidad Nacional del Centro de la Provincia de Buenos Aires. Olavarría.

Stoessel, L. \& Martínez, G. (2014). El proceso de intensificación en la transición pampeano-patagónica oriental. Discusión y perspectivas comparativas con regiones aledañas. Comechingonia. Revista de Arqueología, 18, 65-94. 
